\title{
Frauen in der osteopathischen Praxis
}

Gudrun Wagner

\begin{abstract}
Frau in der osteopathischen Praxis gerecht zu werden, ist ein komplexes Unterfangen. Dieser Beitrag will neue Forschungsergebnisse, biologische und soziale Komponenten sowie osteopathische Zugänge in Kontext zueinander stellen - und so ein spannendes, umfassendes Bild zeichnen, das einerseits zum Nach- und Weiterdenken anregen soll und andererseits auch zu einem osteopathisch vertieften Arbeiten führen darf.
\end{abstract}

\section{Schau, eine Frau!}

Stellen wir uns einmal diesen gar nicht außergewöhnlichen Fall vor: Eine Frau kommt zur Behandlung.

Bis jetzt wird in medizinischen wie osteopathischen Ausbildungen meist am Modell Mann gelehrt, diese „andere“ Anatomie, Physiologie der Frau wird nur in Bezug zu primären und sekundären Geschlechtsmerkmalen vermittelt. Doch es ist wichtig, soziokulturelle Überlegungen und gendermedizinische Forschungsergebnisse in unsere osteopathischen Arbeitsweisen einzubeziehen. Vielleicht können wir so unseren Händen erklären, was diese schon lange spüren: Frau spürt sich anders an als Mann. Was ist also eine Frau? Und wie holen wir unsere Patientin am besten ab?

Wir müssen Frau in ihrem Frau-Sein begreifen - als einen Menschen mit biologischer Grundausstattung in einem sozialen Umfeld. Frau hat also ein Sex, ein biologisches Geschlecht (beruhend auf Genetik, Anatomie, Physiologie und allem Körperlichen) und Frau hat ein Gender, d. h. ein soziokulturelles Geschlecht (Einflüsse kultureller und sozialer gesellschaftlicher Gegebenheiten auf die Geschlechterrolle und die Positionierung in der Gesellschaft), das sich auch potenziell stets weiter verändert.

Biologisch werden gemeinhin 2 Geschlechter unterschieden: weiblich und männlich. Westliche Gesellschaften erkennen auch nur 2 soziale Rollen an, nämlich Mann und Frau. Lässt sich keine Zuordnung zu einem der beiden Geschlechter vornehmen (als Kind), gibt es inzwischen in mehreren Ländern die Möglichkeit, ein unbestimmtes Geschlecht einzutragen (u.a. in Malta, Deutschland, Dänemark, Neuseeland, Indien, Australien, Argentinien). Manche Gesellschaften haben 3 Gender - Männer, Frauen und Berdachen (oder Hirjas oder Xanith) oder kennen den Gender-Status Frauen mit Männerherz, nämlich biologi- sche Frauen, die als weibliche Männer leben, aber sich nicht so verhalten oder kleiden [1,2].

Frau hat ihr biologisches Geschlecht (kein Mann oder Hermaphrodit), ihre Sexualität (hetero-, homo-, bi- oder transsexuell) und ihr Gender (Mädchen, Frau, Männerrolle, Transvestit, Greisin). Im Alltag ist uns Gender so vertraut, dass die Erwartungen, wie Frauen und Männer sich verhalten sollen, gewöhnlich erst bewusst durchbrochen werden müssen, damit wir merken, wie sich Gender konstituiert. Beim Individuum beginnt die Gender-Konstruktion damit, dass es je nachdem, wie die Genitalien bei der Geburt aussehen, einer bestimmten Sex-Kategorie zugeordnet wird (und zweideutige Genitalien werden chirurgisch leider oftmals zu früh weiblich oder männlich vereindeutigt [1]. Aus einer Sex-Kategorie wird durch Namen, Kleidung und andere Gender-Marker ein Gender-Status. Ist ein Gender einmal offensichtlich, sind auch die Umweltreaktionen (inner- und außerfamiliär) beim einen Gender anders als beim anderen, und auch das Kind reagiert auf die unterschiedliche Behandlung, indem es sich anders fühlt und ein anderes Verhalten zeigt. Alle Lebenserfahrungen prägen und erzeugen unterschiedliche Gefühle, unterschiedliches Bewusstsein, unterschiedliche Beziehungen, unterschiedliche Fähigkeiten - eben die Seinsweisen, die wir als weiblich oder männlich bezeichnen - und aus denen die soziale Konstruktion Gender besteht [1]. Jede Frau hat ihr Sex und ihr Gender und kann ihre Biologie und ihr Rollenbild als kongruent erleben oder als widerstreitend. Wie Frau sich selbst in ihrer Geschlechtlichkeit erlebt, biologisch wie soziokulturell, ist entscheidend für ihr Sein, ihren individuellen Gesundheitsbegriff und ihren Lebensplan.

Nun können wir Frau dort, wo sie in diesem Moment ist und sich gesund fühlt, osteopathisch abholen - und nur so können wir uns von einem rein biologischen Zugang 
erfolgreich abwenden und Frau in ihrem Menschsein begreifen.

Aber natürlich ist und hat Frau, unsere Patientin, auch einen Körper. Die seit Jahrzehnten betriebene medizinisch-geschlechtsspezifische Forschung zeigt hier immer mehr Unterschiede auf, sie geht zum Teil weit über das tradierte Bild der Frau als eine zur Reproduktion fähige und deshalb auf diese Organe reduzierbare östrogenreiche Variante Mensch hinaus. Denn nur manche Frauen sind schwanger und auch dann nur eine gewisse Zeit, manche Frauen haben keine Gebärmutter oder keine Eierstöcke, bei manchen Frauen setzt die Menstruation zeitweise aus und andere sind in den Wechseljahren [1] - und doch sind alle Frauen! Menstruation, Milchbildung und Schwangerschaft sind individuelle Erfahrungen des Frauseins [3], nicht aber Determinanten.

\section{Relevantes aus der Forschung}

Einige osteopathisch sehr relevante Ergebnisse der Forschungen, wie hormonelle Zusammenhänge, neurologische Outputs, immunologische Interaktionen, Besonderheiten des Bewegungsapparats und kardiale Fakten, sollen exemplarisch aufgezeigt werden und sind selbstverständlich auf dem Hintergrund einer soziokulturellen Interpretation interessanter zu lesen.

\section{Hormonelle Situation}

Die spezifische hormonelle Situation von Frauen macht sie zu potenziell flexibleren Menschen, die mit größeren Risiken, stärkeren Belastungen und länger währenden Einschränkungen besser umgehen können. Sexualsteroide haben diverse extragenitale Funktionen (Haut, Lungen, Knochen). Östrogene modulieren Hunderte von Proteinen. Exemplarisch sei die Interaktion zwischen weiblichen Sexualsteroiden und dem Herz-Kreislauf-System erwähnt [4].

Die Hormone der Frau schützen ihr Herz. Dieser kurative Effekt ist vor der Menopause gegeben. Östrogene senken den Cholesterinspiegel durch Upregulation der LDL-Rezeptoren und bewirken eine Upregulation der endothelialen Stickstoffmonoxidsynthase, unterdrücken Endothelin und haben einen kalziumantagonistischen Effekt. Dadurch besteht bei der Frau eine ganz andere hämodynamische Situation als beim Mann - eine protektive Vasodilatation während Menstruationzyklus und Schwangerschaft und ein modulierender Einfluss auf den Blutdruck (Schutz vor Hypertonie) und auf die Herzfrequenz (Reduktion von Palpitationen) [5].

\section{Muskel-Ligament-Skelett-System}

Das weibliche Muskel-Ligament-Skelett-System ist mit dem männlichen nicht vergleichbar. Zwar sind die anatomischen Substrate an Zahl identisch, aber nicht in Form und nicht in Funktion, d.h. Artikulationsflächen, bio- mechanische Achsen und Winkel und somit Funktionsgrade sind für Frauen neu zu bestimmen. Neueste Forschungen fordern ein Umdenken, da quasi kein Knochen auf dem anderen bleibt.

Bereits bei der Geburt ist das weibliche Skelett leichter, obwohl es weiter entwickelt ist, und bleibt auch im Durchschnitt um 25\% leichter als das männliche. Dieser leichtere Knochenbau kommt besonders dadurch zustande, dass der Strebenbau der Röhrenknochen zarter ist. Die Rumpfbetonung von Frauen ist mit 38\% der gesamten Körperlänge um 2\% größer als die des Mannes, die Extremitätenbetonung also geringer. Aber nicht nur die Rumpflänge verändert die Körperproportionen, sondern auch die größere Hüftbreite, die kompensatorisch zu einer X-Bein-Stellung führt. Dies bewirkt eine Verlagerung des Körperschwerpunkts nach unten, zudem ist die Pronation des Fußes durch ihre breitere Hüftstellung stärker. Bekannt ist auch, dass sich die tägliche Anpassung des Knochenmetabolismus ebenso unterscheidet wie der Zeitpunkt der höchsten Knochendichte [6]. Am Knie [7-11] besteht ein signifikant größerer Quadrizeps-Winkel zwischen Tuberositas tibiae und Patella. Dadurch läuft die Patella-Führungslinie eher in Valgusform auf die Mittellinie der Kondylen zu, bedingt durch die bei Frauen vermehrt beobachtete Antetorsion des Schenkelhalses. Ferner sind die Femurkondylen mehr trapezförmig und die Vorderseite ist schmäler. Beim Azetabulum weiß man, dass Frauen eine mehr nach anterior orientierte Pfanne haben [12].

\section{Nervensystem}

In der Hirnforschung zeigen Studien, dass Frauen mit beiden Hemisphären ausgeglichen (bilateral) denken und hören. Auch das Sprachzentrum ist rechts- und linksseitig ausgeprägt und nicht nur linksseitig wie bei Männern, wie aktuelle Studien über das Wiedererlernen der Sprache nach apoplektischem Insult belegen. Eine Studie zeigt die gesteigerte Symmetrie in weiblichen Gehirnen, v. a. der grauen Substanz, und hier wiederum von Thalamus, Kleinhirn und sprachassoziierten Regionen [13].

Die nach außen sichtbaren und analysierbaren Outputs des Nervensystems haben auch eine innere Komponente. Die Entwicklung und Differenzierung von Zellen kann sowohl durch zellintrinsische (zellautonome) als auch durch extrinsische Signale aus der Umgebung reguliert werden, und bestimmte Hirnregionen zeigen geschlechtsspezifische Besonderheiten, insbesondere Basalganglien, limbisches System und Neokortex, wo Motorik, Emotionen oder kognitive Funktionen gesteuert werden.

Bei der Untersuchung der geschlechtsspezifischen Differenzierung verschiedener spezifischer Nervenzelltypen, wie der dopaminergen Neuronen aus dem Mittelhirn und dem Hypothalamus, wurde gezeigt, dass diese Neurone morphologische und funktionelle Geschlechtsunter- 
schiede entwickeln. Daraus wurde die Hypothese entwickelt, dass Neurone ihr genetisches Geschlecht auch zellautonom realisieren können; die geschlechtsspezifische Differenzierung des Gehirns wäre so als Ergebnis einer Interaktion von zellextrinsischer Regulation durch Steroidhormone und zellintrinsischer Genaktivierung zu verstehen [14]. Weitere neuroanatomische Besonderheiten weisen die Strukturen auf, in denen eine Steroidgenese (Progesteron und Metaboliten) stattfindet: Amygdala, Hippokampus, Retina und Neuronen in Bulbus olfactorius, Thalamus, Hypothalamus und Corpus striatum [15].

\section{Immunsystem}

Ein wesentlicher Unterschied des weiblichen Immunsystems zum männlichen ist seine Flexibilität. Die Immunsituation verändert sich zyklusbedingt (Ovulation) wie auch durch Schwangerschaft und nach der Menopause. Östradiol hat eine hemmende und stimulierende Wirkung auf das immunologische Netzwerk, Progesteron wirkt stark immunmodulierend. Prä- und periovulatorisch kommt es - östrogenbedingt - zu einer enormen Steigerung der immunologischen Aktivität, die in der Lutealphase - progesterondominiert - rasch wieder sinkt. Diese immunregulativen Mechanismen kommen nicht nur im Endometrium zum Tragen, sondern spielen sich im gesamten weiblichen Körper, im besonderen aber im Bereich der Schleimhäute, ab. Die immunologische Potenz einer Frau ist also in der 1. Zyklusphase größer als in der 2. [16]. Während einer Schwangerschaft entwickelt sich das Immunsystem weg von der zellmediierten Immunität und hin zu mehr humoral induzierter Immunität.

In den Wechseljahren bzw. nach dem Eintritt der Menopause, sobald die Östrogenproduktion reduziert ist oder sistiert (auch bei künstlicher Menopause), verringert sich die Menge an zirkulierenden Antikörpern und die Immunantwort wird langsamer. Diese immunologische Alterung lässt Frauen trotzdem im Alter gesünder sein und ihre Lebenserwartung ist länger.

Die Haut z. B. moduliert durch das komplexe Zusammenspiel von Östrogenen und Androgenen das Immunsystem, die Dicke der Epidermis und Dermis. So verändern sich u.a. der pH-Wert, die Qualität der Wundheilung und die Wahrscheinlichkeit eine Autoimmunerkrankung zu entwickeln [17]. Das gesamte Mikrobiom des Darms und damit das Immunsystem ist geschlechtsspezifisch [18].

\section{Die Diagnose-Diva ${ }^{\circledR}$}

Frau-Sein allein (das wissen Sie jetzt) ist nicht der Grund für den Besuch bei der Osteopathin und es ist auch keine Diagnose. Als Osteopathinnen begreifen wir unser Gegenüber als Ganzes, versuchen Frau in ihrem Lebenskontext zu verstehen und zu erfassen, um sie wertschätzend dort abholen zu können, wo sie steht oder wo sie hin- strebt. Die besondere Herausforderung besteht darin, ein Gleichgewicht zwischen allgemeinen und frauenspezifischen Befunden zu finden, ohne den Menschen aus den Augen zu verlieren, d.h. ohne auf frauenspezifische und Gender-Merkmale im Übermaß oder reduktionistisch einzugehen. Wir wollen also einen Menschen osteopathisch behandeln, eine Frau kennen- und verstehen lernen und letztlich auch diagnostizieren, eine Frau, die mit spezifischen, aber nicht unbedingt frauenspezifischen Anliegen zu uns kommt.

Die Diagnose-Diva ${ }^{\odot}(\triangleright$ Abb. 1) zeigt die Komplexität der Diagnose auf und stellt eine Übersicht der diagnostischen Kriterien dar. Die unten gewählte Reihenfolge ist nicht nach Wichtigkeit geordnet und veränderbar.

\section{Hormonelles Gleichgewicht}

Wie ist das hormonelle Gleichgewicht? Wie reagiert das Gewebe auf die Hormone? Sind die einzelnen Organe miteinander in Kommunikation? Gibt es Restriktionen in der Hormonachse? Man prüft also die Motilität der angesprochenen Strukturen, auch ihre Mobilität und die Gewebequalität sowie die Responsibilität des Gewebes auf Inputs und die Kapazität der Outputs.

\section{Nervensystem}

Ist das Nervensystem in seinen Bewegungen frei? Hat es einen Fokus? Wie ist seine neurotrophe Gewichtung? Reagiert es im gleichen Maße auf innen und außen? Wie ist das Gleichgewicht zwischen Sympathikus und Parasympathikus?

\section{Immunsystem}

In welcher Situation befindet sich das Immunsystem gerade? Zeigt es eine Abwehr von Infektionen, Hyperaktivität, Restriktionen? Sind die immunologischen Organe in Kommunikation untereinander? Sind die Lymphwege frei? Ist das Immunsystem des Darms aktiv? In die Überprüfung des Immunsystems sollten neben der Überprüfung der Motilität von Milz, Thymus, der Evaluierung des Waldeyer-Rachenringes, der Peyer-Plaques und der Cysterna chyli auch die Prüfung der großen Lymphbahnen und Lymphknoten miteingeschlossen sein. Die Gesamtsituation, z. B. allergene Disposition, Unverträglichkeiten, akute oder chronische Infektion, autoimmunologische Prozesse, Tumorerkrankungen, medikamentöse Immunsuppression, sollte die Ausgangsbasis für weitere Diagnose- wie Behandlungsschritte sein.

\section{Interner Informationsaustausch}

Sind das Hormon-, das Immun- und das Nervensystem im Gleichgewicht und in Kommunikation miteinander? Reagiert das eine System adäquat auf die Informationen der anderen? 


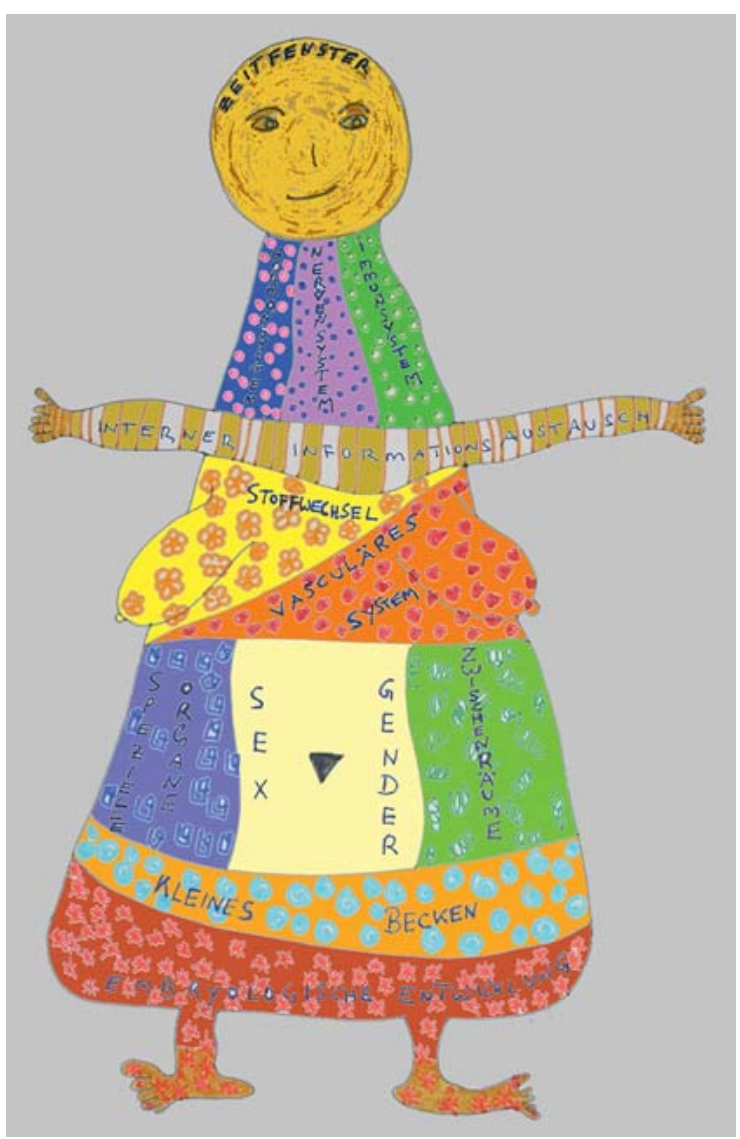

- Abb. 1 Diagnose-Diva ${ }^{\circ}$. (aus: [19])

\section{Vaskuläres System}

Wie ist die Versorgung, die Vitalität des Körpers? Ist das Kreislaufsystem belastbar? Besteht ein Gleichgewicht zwischen sympathikotoner und parasympathikotoner Beeinflussung? Gibt es lokale Permeabilitätsstörungen? Bilden das arterielle und das venöse System eine Einheit [20]?

\section{Stoffwechsellage}

Ist die Stoffwechsellage anabol oder katabol? Gibt es diabetische oder andere den Stoffwechsel bestimmende Tendenzen? Die allgemeine Stoffwechselsituation gibt Aufschluss über etwaige bereits oben erwähnte Einflüsse bzw. Zustände (Nerven-, Immun-, Hormonsystem) und zeigt die potenzielle Tendenz momentaner therapeutischer Wirkungen auf.

\section{Zeitfenster}

Bei jeder Behandlung öffnet sich das Fenster neu. In welchem Lebensabschnitt befindet sich Frau? Wie alt ist sie biologisch? Wie alt fühlt sie sich? Welche Ziele hatte sie und welche hat sie? Wo ist Frau im Zyklus? Hat Frau Zyklus? Welche Zeitparameter hat ihr Zyklus? Ist sie in der Menopause? Ist sie schwanger?

\section{Sex - Gender}

Kann ich mein Gegenüber überhaupt begreifen, ohne sie nicht kennenzulernen?

\section{Kleines Becken}

Das kleine Becken bildet und begrenzt den Rahmen, in dem sich Ausscheidungs- und Fortpflanzungsstrukturen befinden und mit dem Außen in Kontakt kommen. Der Diagnostik dieser Organe sollte immer zuerst eine Beurteilung des Rahmens vorangehen. Wie ist der Raum beschaffen, in dem diese Organe wohnen? Wie elastisch kann der Rahmen sein? Die Gesamtheit des Rahmens kann in seinem fluiden Anteil, in seinem Potenzial zur Flexibilät, also Anpassung an intraabdominale Druckschwankungen, Veränderungen der inneren Gegebenheiten, Positionsänderungen, diagnostiziert werden.

Um die Organe letztendlich diagnostizieren zu können, ist es wichtig, sich zu veranschaulichen, dass die Organe des kleinen Beckens nicht nur in diesem Rahmen, sondern auch in kleinen Extrazimmern wohnen, die ihnen Raum geben, ihre Mobilität zu den Nachbarstrukturen ermöglichen und ihre Ausdehnungsvariationen erleichtern. Diese Zwischenräume entstehen meist durch Peritonealfalten. Ein wichtiges diagnostisches Kriterium ist die freie Bewegung und Verschieblichkeit der einzelnen Räume bzw. die Bewegungsfreiheit der Bindegewebszüge. Mit einem anterior-posterioren Händekontakt kann man sowohl die 4 transversalen als auch die 3 sagittalen Räume prüfen - auf Mobilität, Elastizität, Raumschaffung, Raumerhaltung und Spannung.

\section{(Geschlechts-)spezifische Organe}

Die Gebärmutter ist ein extrem flexibles, mobiles Organ, das mehr in seinen Aufhängungen schwebt als sich abstützt. Die Diagnostik betrifft also wieder den Raum, in dem der Uterus wohnt und wie er darin gehalten wird. Wie ein Schmetterling schwebt die Gebärmutter, ihren Korpus mit der Zervix auf die Vagina abstützend, mittels ihrer Flügel zwischen den Beckenwänden.

Beim Ovar ist zu beachten, dass eines stets anders aussieht als das andere und es unterschiedlich groß ist. Zuerst ist es klein und glatt, ab der Menarche wird es größer und bleibt groß bis zur Menopause. Durch die Eisprünge wird es zunehmend vernarbt. Zyklusabhängig ist es mehr oder weniger stark durchblutet. Nach der Menopause verkleinert es sich wieder, bis es nach dem Klimakterium nur mehr sehr klein ist. Demzufolge braucht es unterschiedlich viel Raum und der Spannungszustand der Ligamente ist veränderlich. Das Zusammenwirken mit den Fimbrien der Tube ist ein wichtiger diagnostischer Hinweis bei unerfülttem Kinderwunsch und wiederholten Eileiterschwangerschaften. Die gleichzeitige Diagnostik des rechten und linken Ovars macht funktionale Läsionen deutlich. 
Die Mesosalpinx, eine Bauchfellduplikatur aus beiden Blättern des Lig. latum uteri, die zur Beckenwand zieht und zwischen Lig. teres uteri und Lig. ovarium propium liegt, geht um die gesamte Tuba uterina, deren Kriterium ihrer Beweglichkeit eben die spannungsfreie Mesosalpinx ist.

Bei der Diagnostik der weiblichen Brust ist es wichtig, zwischen den elementaren Bestandteilen - Milchgänge, Endknospen, im Gangsystem liegenden wachstumsbereiten undifferenzierten embryonalen Zellen, die neue Endknospen bei Schwangerschaften bilden können - und dem konstituierendem Fettgewebe zu unterscheiden. In der Pubertät entwickeln sich durch die weiblichen Steroidhormone infolge des Wachstums des subkutanen Fettgewebes die Brüste. In jedem Zyklus kommt es zu einem Wachstumsschub im Gangsystem durch Östrogene. Progesteron induziert eine abortive Differenzierung der Endknospen. Eine Diagnose der Brüste muss immer den momentanen Zyklus, eine mögliche Schwangerschaft, vorbestehende Schwangerschaften oder Hormontherapien miteinbeziehen.

\section{Schau, schau!}

Stellen wir uns den spannenden Fall vor: Eine Frau kommt zur Behandlung. Zurecht sind sie aufgeregt: Wie erlebt sich diese Frau, wie wurde diese Frau zu so einer Frau, wo befindet sie sich, wohin will sie? Ein Meer an Vielfalt tut sich auf. Vor diesem soziokulturellen Hintergrund entdecken Sie Ihre Patientin und treffen sie in ihrem gesunden Potenzial. Und so offenbart sich dann auch Ihren osteopathischen Händen ihr Körper mit all' seinen Geheimnissen, geschlechtsspezifischen wie allgemein menschlichen. Und am Ende der Behandlung können Sie sich hoffentlich staunend zurücklehnen und denken: Schau, schau - eine Frau!

\section{Über die Autoren}

\section{Gudrun Wagner}

ist Ärztin, Osteopathin, Kinderosteopathin und Künstlerin (Studium der Bildhauerei). Sie ist im Vorstand des Osteopathischen Zentrums für Kinder (OZK) in Wien. Sie lehrt in Deutschland, Italien und der Schweiz und hat diverse Publikationen veröffentlicht (Zeitschriften, Fachbuchbeiträge).

\section{Korrespondenzadresse}

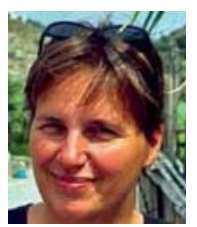

Dr. Gudrun Wagner, MSc., D.O., DPO

Piaristengasse $13 / 3$

1080 Wien

Österreich

gudrunwagner@a1.net

www.ozk.at

\section{Literatur}

[1] Lorber Judith. Gender-Paradoxien. Opladen: Leske + Budrich; 1999

[2] Amadiume I. Male daughters, female husbands. Gender and sex in an african society. London: Zed Books; 1999

[3] Levesque-Lopman L. Claiming reality. Phenomenology and women's experience. Totowa: Rowman \& Littlefield; 1988

[4] Radhika N et al. Cardiovascular Actions of Selective Estrogen Receptor Modulators and Phytoestrogens. Preventive Cardiology 2004; 7: 2

[5] Basile JN. Analysis of recent papers in hypertension. Journal of Clinical Hypertension 2002; 1: 47-51

[6] Legato M. Vortrag zur Gender-Medizin; Wien 2007

[7] Chin KR et al. Intraoperative measurements of male and female distal femurs during primary total knee arthroplasty. J Knee Surg 2002; 15 (4): 213-217

[8] Hitt $\mathrm{K}$ et al. Anthropometric measurements of the human knee: correlation to the sizing of current knee arthroplasty systems. J Bone Joint Surg 2003; 85: 115-122

[9] Hsu RWW et al. Normal axial alignment of the lower extremity and load bearing distribution at the knee. Clin Orthop 1990; 255: $215-227$

[10] Woodland LH, Francis RS. Parameters and comparisons of the quadriceps angle of college-aged men and women in the supine and standing positions. Am J Sports Medicine 1992; 20: 208-211

[11] Mahfouz $\mathrm{M}$ et al. Analysis of variation of adult femora using sex specific statistical atlases. Presented at: Computer Methods in Biomechanics and Biomedical Engineering Conference, Antibes, 2006

[12] Musielak B, Rychlik M, Jóźwiak M. Sexual Dimorphism of Acetabular Anatomy Based on Three-dimensional Computed Tomography Image of Pelvises. Orthop Traumatol Rehabil 2016;18 (5): 451-459

[13] Núñez C et al. A large-scale study on the effects of sex on gray matter asymmetry. Brain Struct Funct 2017; 1863-2653: 111

[14] Schmitz S. Über den Geschlechtsdeterminismus in der Hirnforschung - Ansätze zu seiner Dekonstruktion. Forum Wissenschaft 2004; 21 (4): 9-13

[15] Pilgrim C, Beyer C, Reisert I. The effects of sex and sex hormones on the development of dopaminergic neurons. Austin: R.G. Landes; 1999: 75-86

[16] Huber J. Frauenspezifische Medizin. Wien: Maudrich; 2005

[17] Dao H, Kazin RA. Gender differences in skin: a review of the literature. Gend Med 2007; 4 (4): 308-328

[18] Fransen F et al. The Impact of Gut Microbiota on GenderSpecific Differences in Immunity. Front Immunol 2017; 8: 745

[19] Riedl KH, Schleupen A. Osteopathie in der Frauenheilkunde. München: Elsevier; 2010

[20] Liem T, Dobler TK, Puylaert M. Leitfaden viszerale Osteopathie. München: Elsevier; 2005

Bibliografie

DOI https://doi.org/10.1055/s-0043-121261

DO - Deutsche Zeitschrift für Osteopathie 2018; 16: 24-28

(c) Georg Thieme Verlag KG Stuttgart · New York ISSN 1610-5044 